\title{
Improving Gaussianity of EMG Envelope for Myoelectric Robot Arm Control
}

\author{
SANDRA M'ARQUEZ-FIGUEROA, YURIY S. SHMALIY, OSCAR IBARRA-MANZANO \\ Dept. of Electronics Engineering Universidad de Guanajuato Salamanca, MEXICO
}

\begin{abstract}
Several methods have been developed in biomedical signal processing to extract the envelope and features of electromyography (EMG) signals and predict human motion. Also, efforts were made to use this information to improve the interaction of a human body and artificial protheses. The main operations here are envelope acquiring, artifacts filtering, estimate smoothing, EMG value standardizing, feature classifying, and motion recognizing. In this paper, we employ EMG data to extract the envelope with a highest Gaussianity using the rectified signal, where we deal with the absolute EMG signals so that all values become positive. First, we remove artifacts from EMG data by using filters such as the Kalman filter (KF), H1 filter, unbiased finite impulse response (UFIR) filter, and the $\mathrm{cKF}, \mathrm{cH} 1$ filter, and cUFIR filter modified for colored measurement noise. Next, we standardize the EMG envelope and improve the Gaussianity. Finally, we extract the EMG signal features to provide an accurate prediction.
\end{abstract}

KeyWords: EMG envelope, Gaussianity, filtering, features, classification, artifacts

Received: March 12, 2021. Revised: July 15, 2021. Accepted: July 28, 2021. Published: August 8, 2021.

\section{Introduction}

Many investigations have been carried out for diverse electromyography (EMG) raw databases to extract features with a highest accuracy using several standard metrics and different methods [1]. The EMG signal is a register of the electrical activity of the muscles with positive and negative deflections. The classification methods allow analyzing the performance of the obtained data and are often combined with different technologies to improve the envelope Gaussianity and increase the accuracy in the extracted features. On this way, it is required to remove noise using a good quality digital filter [2], [3]. Solutions can be found in bio-electronics [4], bio-mechanics [5], [6], and bio-robotics [7], where the EMG frequency and amplitude are employed to identify motions.

The EMG envelope is extracted using a rectified signal, where the negative values are converted to be positive [8], [9]. Some researchers achieve an efficient noise reduction using the reflected envelope. A drawback is that it often causes unacceptable bias errors [10], [11] and does not prevent spikes. Therefore, filtering technics are involved. In [12], the envelope is improved using the Savitsky-Golay smoother combined with a low-pass filter that, however, introduces a time-delay lag. In this investigation, we use the advanced filters [3], which allow for a high accuracy is the artifacts removal and improving the Gaussianity. We employ the NinaPro database with a resolution over a $10 \mathrm{mV}$ range, which contains the records of the upper limbs of 27 intact subjects while performing 52 fingers, hand, and wrist movements of interest [13]. All tests of synthetic data are provided using special software. The noise value is determined by either measurements or computation [14]-[17].

\section{EMG Signal Envelope Problem}

In this section, we consider the EMG envelope extraction problem and discuss the kinematic model of a robot arm.

\subsection{EMG Signal Envelope}

We need to extract the EMG envelope because the EMG signal is bipolar to mean that it has both positive and negative values with respect to the base line around zero. The signal rectification is used to form the absolute EMG signal. After rectified, all values become positive with a number of spikes. The spikes are required to be eliminated using smoothing algorithms. In our study, we approximate the EMG envelope with a $(K+1)$-degree polynomial, where $K$ is the number of the envelope states, using the Taylor series expansion, in which case the system matrix is given by [18]

$$
F=\left[\begin{array}{ccccc}
1 & \tau & \frac{\tau^{2}}{2} & \cdots & \frac{\tau^{K-1}}{(K-1) !} \\
0 & 1 & \tau & \cdots & \frac{\tau^{K-2}}{(K-2) !} \\
0 & 0 & 1 & \cdots & \frac{\tau^{K-3}}{(K-3) !} \\
\vdots & \vdots & \vdots & \ddots & \vdots \\
0 & 0 & 0 & \cdots & 1
\end{array}\right] \in \mathbb{R}^{K \times K} .
$$

The problem arises of identifying the best processing algorithm achieving the highest estimation accuracy, in order to increase the accuracy of features extraction and the best envelope Gaussianity. To this end, we make a comparison of the traditional filters and advanced filters considering ripples in the envelope as the colored measurement noise (CMN). Thereby, our report is better geared towards the monitoring and evaluating the envelope trends. In addition, we use two additional algorithms to smooth the envelope at the output of each of the filters. 


\subsection{Kinematic model of Robot Arm}

The system dynamics is understood when we have an adequate knowledge of its functioning. We are now consider a structure of the robotic arm that is constituted with links, joints, and handlings, where the inclination angle and the length of the first and second link are given by $\Phi_{1}, l_{1}$, $\Phi_{2}$, and $l_{2}$. A system will recognize the EMG motion at a millimeter accurate. The kinematic model of a robotic arm gives the related speed $\dot{h}_{(X, Z)}$ of an interesting point to the angular speed $\dot{\Phi}$ for each motor located in the joints. We obtain this relationship by using the Jacobian matrix. To have a mathematical model of the two degrees of freedom (2DOF) robotic arm, we consider the plane perpendicular to the reference $X$ and $Z$. From the Creeper we create the following mathematical model,

$$
\begin{aligned}
h_{X}= & X_{1}+X_{2}, \\
h_{Z}= & A+Z_{1}+Z_{2}, \\
h_{Z}= & A+Z_{1}, \\
h_{X}= & l_{1} \cos \left(\Phi_{1}\right)+l_{2} \cos \left(\Phi_{1}+\Phi_{2}\right), \\
h_{Z}= & A+l_{1} \sin \left(\Phi_{1}\right)+l_{2} \sin \left(\Phi_{1}+\Phi_{2}\right), \\
\dot{h}_{X}= & \left(-l_{1} \sin \left(\Phi_{1}\right)-l_{2} \sin \left(\Phi_{1}+\Phi_{2}\right)\right) \dot{\Phi_{1}} \\
& -\left(l_{2} \sin \left(\Phi_{1}+\Phi_{2}\right)\right) \dot{\Phi_{2}}, \\
\dot{h}_{Z}= & \left(l_{1} \cos \left(\Phi_{1}\right)-l_{2} \cos \left(\Phi_{1}+\Phi_{2}\right)\right) \dot{\Phi_{1}} \\
& +\left(l_{2} \cos \left(\Phi_{1}+\Phi_{2}\right)\right) \dot{\Phi_{2}},
\end{aligned}
$$

where $\Phi_{1}$ and $\Phi_{2}$ are the angle of inclination of each link Kudo, $l_{1}$ and $l_{2}$ are the lengths of each of the links, $\dot{h}_{x}$ and $\dot{h_{y}}$ are the angular speeds, $\dot{\Phi_{1}}$ and $\dot{\Phi_{2}}$ are the speeds of each the motors located in the links, while $\ddot{\Phi}_{1}$ and $\ddot{\Phi}_{2}$ give the accelerations. The model is used to control the motor position according to the reference position and thus to bring the application to control the motors of a prosthesis. Next, the equations of the dynamic system will be be expressed as a mathematical model with a set of variable inputs.

\section{Materials and Methods}

\subsection{State-Space Model of EMG Envelope}

The EMG envelope is represented in discrete-time index $i$ with the $K$-state-space polynomial model [18] assuming the Gauss-Markov CMN $v_{i}$ as [19]

$$
\begin{aligned}
x_{i} & =F x_{i-1}+B w_{i}, \\
y_{i} & =H x_{i}+v_{i}, \\
v_{i} & =\psi_{i} v_{i-1}+\xi_{i},
\end{aligned}
$$

where

- $x_{i}$ is the state vector.

- $y_{i}$ is the scalar observation of the envelope first state.

- $F$ is the system matrix.

- $H$ is the observation matrix.

- $\psi_{i}$ is the scalar coloredness factor.

- $B$ is the system noise matrix.

- $w_{i}$ is the envelope noise.
The observation matrix is given by $H=\left[\begin{array}{llll}1 & 0 & \ldots\end{array}\right] \epsilon$ $\mathbb{R}^{1 \times K}$ and the system noise matrix is $B \in \mathbb{R}^{K \times P}$. Matrix $B$ projects the envelope noise $w_{i} \in \mathbb{R}^{P}$ into $x_{i}, \psi_{i}<1$ is supposed to be known at each $i$ and such that noise $v_{i}$ is stationary. Namely, by $\psi_{i}=0$, noise $v_{i}$ becomes white Gaussian, as required by optimal estimators.

Because the EMG envelope noise $w_{i}$ is generally unknown, we will think that it has zero mean with uncertain both the statistics and distribution. However, to run the KF, we will consider $w_{i}$ as zero mean and white Gaussian, $w_{i} \sim$ $\mathcal{N}(0, Q) \in \mathbb{R}^{P}$, with the covariance $E\left\{w_{i} w_{j}^{T}\right\}=Q \delta_{i-j}$, where $\delta_{i}$ is a Kroneker symbol, which has unknown entries. Noise $\xi_{i}$ is zero mean and white Gaussian, $\xi_{i} \sim \mathcal{N}\left(0, \sigma_{i}^{2}\right)$, with the variance $E\left\{\xi_{i}^{2}\right\}=R=\sigma_{\xi}^{2}$ and the property $E\left\{w_{i} \xi_{j}\right\}=0$ for all $i$ and $j$.

We assume that the estimate $\hat{x}_{i} \triangleq \hat{x}_{i \mid i}$ of $x_{i}$ under the intensive ripples in the envelope will range closer to the desired envelope under the supposedly CMN. Therefore, below we will develope two possible linear approaches to shape the envelope: the KF, which requires all information about the initial values and white Gaussian noise, and the UFIR filter, which completely ignores these requirements and is thus more robust [21]. We will also consider the $H_{\infty}$ filter and call the modified solutions as $\mathrm{cKF}, \mathrm{c} H_{\infty}$ filter, and cUFIR filter.

\section{2 cKF Algorith/m}

To apply the KF to (11)-(13), one can follow [21], consider a new observation $z_{i}$ as measurement differences, and write

$$
\begin{aligned}
z_{i} & =y_{i}-\psi y_{i-1}, \\
& =H x_{i}+v_{i}-\psi H x_{i-1}-\psi v_{i-1} .
\end{aligned}
$$

By taking $x_{i-1}$ from (11) and $v_{i-1}$ from (13), a new observation can be written as

$$
z_{i}=D x_{i}+\bar{v}_{i},
$$

where $D=H-\Gamma, \Gamma=\psi H F^{-1}$, and

$$
\bar{v}_{n}=\Gamma B w_{i}+\xi_{i}
$$

is white Gaussian scalar noise with the properties,

$$
\begin{aligned}
E\left\{\bar{v}_{i}^{2}\right\} & =\Gamma \Phi+R=\Gamma \Phi+\sigma_{\xi}^{2}, \\
E\left\{\bar{v}_{n} w_{i}^{T}\right\} & =\Gamma B Q
\end{aligned}
$$

where the weighted matrix $Q$ is

$$
\Phi=B Q B^{T} \Gamma^{T}
$$

The modified state-space model (11) and (15) has now timecorrelated and white $w_{i}$ and $\bar{v}_{i}$ and the KF can be applied, if to derive the optimal bias correction gain taking into account 
the correlation. For given $y_{i}, \hat{x}_{0}, P_{0}, Q, R, \psi$, and CMN, the cKF algorithm becomes

$$
\begin{aligned}
z_{i} & =y_{i}-\psi y_{i-1}, \\
P_{i}^{-} & =F P_{i-1} F^{T}+B Q B^{T}, \\
S_{i} & =D P_{i}^{-} D^{T}+R+H \Phi+\Phi^{T} D^{T} \\
K_{i} & =\left(P_{i}^{-} D^{T}+\Phi\right) S_{i}^{-1}, \\
\hat{x}_{i}^{-} & =F \hat{x}_{i-1}, \\
\hat{x}_{i} & =\hat{x}_{i}^{-}+K_{i}\left(z_{i}-D \hat{x}_{i}^{-}\right), \\
P_{i} & =\left(I-K_{i} D\right) P_{i}^{-}-K_{i} \Phi^{T}
\end{aligned}
$$

and, by $\psi=0$ and $\Phi=0$, it becomes the standard KF.

\section{$3.3 \mathbf{~ c H} \infty$ Algorithm}

The $H_{\infty}$ filter has been derived based on the game theory in [20] and represented in [21] as

$$
\begin{aligned}
\bar{P}_{i} & =\bar{P}_{i}^{-}\left(I-\theta \bar{S}_{i} \bar{P}_{i}^{-}+H^{T} \bar{R}^{-1} H \bar{P}_{i}^{-}\right)^{-1}, \\
K_{i}^{\infty} & =\bar{P}_{i} H^{T} \bar{R}^{-1} \\
\hat{x}_{i} & =F \hat{x}_{i-1}+K_{i}^{\infty}\left(y_{i}-H F \hat{x}_{i-1}\right) \\
P_{i+1}^{-} & =F \bar{P}_{i} F^{T}+\bar{Q}
\end{aligned}
$$

where the symmetric positive definite matrices $\bar{P}_{0}, \bar{Q}$, and $\bar{R}$ must be chosen by the designer to have different meanings than in the $\mathrm{KF}$ and $\bar{P}_{1}^{-}$can be computed as $\bar{P}_{1}^{-}=F P_{0} F^{T}+Q$ [21]. Matrix $\bar{S}_{i}$ is constrained by a positive definite matrix $\left(\bar{P}_{i}^{-}\right)^{-1}-\theta S_{i}+H^{T} \bar{R}^{-1} H>0$ to keep (27) positive definite. To weight all error components equally, one must set $S_{i}=I$. A scalar bound $\theta>0$ must be small enough and, for Gaussian noise with no disturbances, $\theta=0$ makes the $H_{\infty}$ filter KF. For the $\mathrm{c} H_{\infty}$ filter, a new observation can be represented in discrete-time state-space as in (14).

\section{4 cUFIR Filtering Algorithm}

Because the UFIR filter does not require any information about noise, except for the zero mean assumption, it is more suitable for EMG signals. To provide a near optimal estimate, this filter requires an averaging horizon $[m, i]$ of $N$ points, from $m=i-N+1$ to $i$, to be optimal $N_{\mathrm{opt}}$ in the MSE sense . Since $w_{i}$ and $\bar{v}_{i}$ are both zero mean and their correlation does not produce bias, then it follows that the UFIR filter can be applied directly to (11) and (15), unlike the KF.

The cUFIR algorithm operates as follows. Given $N, y_{i}$, and $\psi$, one must set $i=N-1, N, \ldots, m=i-N+1$, and $s=i-N+K$ and compute the initial values $G_{s}=$ $\left(C_{m, s}^{T} C_{m, s}\right)^{-1}$ and $\bar{x}_{s}=G_{s} C_{m, s}^{T} Y_{m, s}$ in short batch forms via $Y_{m, s}=\left[\begin{array}{lll}y_{m} & \ldots & y_{s}\end{array}\right]^{T}$ and

$$
C_{m, s}=\left[\begin{array}{c}
D F^{-(K-1)} \\
\vdots \\
D F^{-1} \\
D
\end{array}\right] .
$$

Provided the initial values at $s$, the iteratively updated values appear for $l=s+1, \ldots, n$ using the recursions

$$
\begin{aligned}
z_{l} & =y_{l}-\psi y_{l-1}, \\
G_{l} & =\left[D^{T} D+\left(F G_{l-1} F^{T}\right)^{-1}\right]^{-1}, \\
K_{l} & =G_{l} D^{T} \\
\bar{x}_{l}^{-} & =F \bar{x}_{l-1} \\
\bar{x}_{l} & =\bar{x}_{l}^{-}+K_{l}\left(z_{l}-D \bar{x}_{l}^{-}\right),
\end{aligned}
$$

and the output estimate $\hat{x}_{i}=\bar{x}_{i}$ is taken when $l=i$. It also follows that, by $\psi=0$, the cUFIR algorithm becomes the standard UFIR filter.

The error covariance of the UFIR filter can be computed approximately by the KF error covariance if to replace the Kalman gain $K_{i}$ with $G_{i} D^{T}$ [22]. That yields

$$
\begin{aligned}
P_{i}= & \left(I-G_{i} D^{T} D\right) P_{n}^{-}\left(I-G_{i} D^{T} D\right)^{T} \\
& +G_{i} D^{T}(\Gamma \Phi+R) D G_{i} \\
& -2\left(I-G_{i} D^{T} D\right) \Phi D G_{i} \\
= & P_{i}^{-}-2\left(P_{i}^{-} D^{T}+\Phi\right) D G_{i}+G_{i} D^{T} S_{i} D G_{i} \\
= & P_{i}^{-}-\left(2 P_{i}^{-} D^{T}+2 \Phi+G_{i} D^{T} S_{i}\right) D G_{i},
\end{aligned}
$$

where $P_{i}^{-}$is given by (21) and $S_{i}$ by (22). Note that the cUFIR algorithm does not require $P_{i}$, although the recursion (37) can be included to for any purposes.

\section{Application}

In this section, we apply the cKF, $\mathrm{c} H_{\infty}$, and cUFIR algorithms to three types of movements and compare to the $\mathrm{KF}, H_{\infty}$, and UFIR algorithms. We will consider the EMG signal envelope extracted with the rectified signal, in which case only better denoising of the envelope is required. Next, we will compare features provided by the extracted envelopes.

For all EMG data, we specify model (11)-(13) with two states, $K=2$, and matrices

$$
F=\left[\begin{array}{ll}
1 & \tau \\
0 & 1
\end{array}\right], B=\left[\begin{array}{c}
\frac{\tau^{2}}{2} \\
\tau
\end{array}\right], H=\left[\begin{array}{ll}
1 & 0
\end{array}\right] .
$$

\subsection{Smoothed EMG Envelope}

Since no information about noise is provided, we tune filters to produce consistent estimates with minimal variations about the desired smooth envelope and insignificant time-delays. For the UFIR filter, we experimentally measure $N_{\text {opr }}=170$, which means that data are highly oversampled. To tune the $\mathrm{KF}$, we suppose that the data noise has the standard deviation of $\sigma_{\xi}=50 \mu \mathrm{V}$ and set $\sigma_{w}=0.1 \mathrm{~V} / \mathrm{s}^{2}$ for the KF estimate to be consistent to the UFIR estimate. For the $H_{\infty}$ filter, we consider the case of $S_{n}=I$. and $\theta=1.0 e^{-6}$. We then run the filters and arrive at estimates shown in Fig. $1 \mathrm{~b}$ and Fig. 2b. As can be seen, all filters shape the envelope much better and consistently and there is no essential time-delays, as required. Thus letting the researchers to recognize the features of interest, we notice that methods of optimal filtering can be used to provide a more efficient shaping of the EMG signal envelope than by the rectification. Even so, it is seen that the envelope is still corrupted by multiple excursions. 
In this case, a surface EMG signal is collected from the basic movements of fingers. In Fig. 1a and Fig. 2a, we show the waveforms of such signals, which is a part of database "S14_A1_E3" observed in a time span of $(0 \ldots 0.851) \mathrm{s}$.

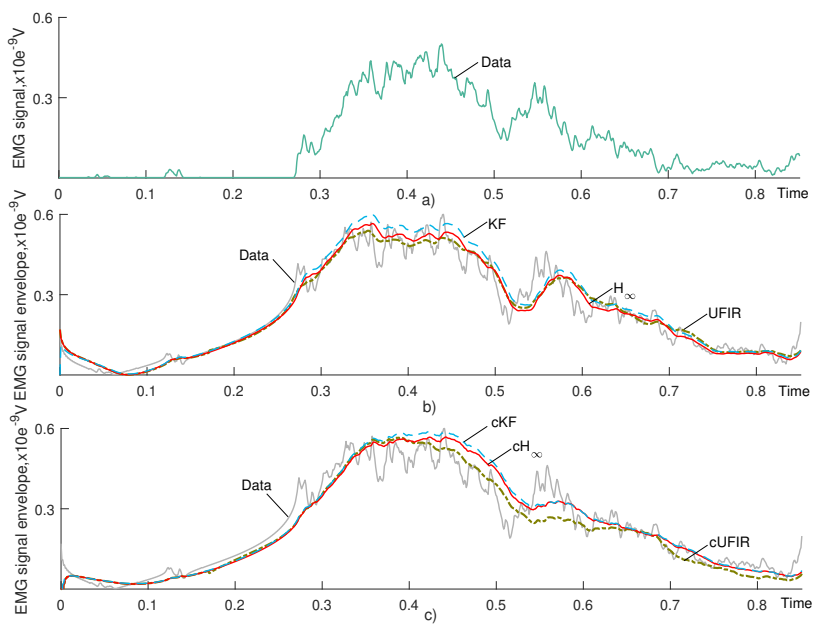

Fig. 1. A part of the EMG signal available from [25]: (a) waveform in the raw state. (b) envelope (data) obtained through the rectificed signal (solid) and its UFIR (dash-dotted), KF (bold), and $H_{\infty}$ (dashed) estimates. (c) cUFIR (dash-dotted), cKF (bold), and $\mathrm{cH}_{\infty}$ (dashed line) estimates.

To suppress the excursions, we next tune the $\mathrm{cKF}, \mathrm{c} H_{\infty}$, and cUFIR algorithms for $\psi=0.65$ and $\bar{N}_{\text {opt }}=140$. The results are shown in Fig. 1c and Fig. 2c. Even a quick look at these figures reveals that many easily seen variations are removed from the envelope, which definitely looks more smoothed. We thus conclude that the Gauss-Markov interpretation of variations and excursions in the EMG signal envelope is useful for better envelope shaping.

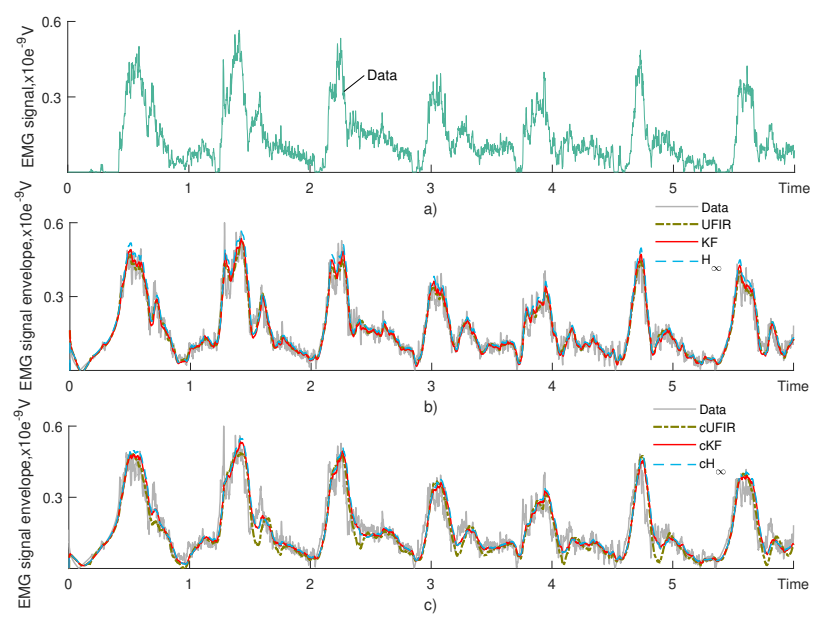

Fig. 2. A part of the EMG signal (0...6)s available from [25]: (a) waveform in the raw state. (b) envelope (data) obtained through therectificed signal (solid) and its UFIR (dash-dotted), KF (bold), and $H_{\infty}$ (dashed) estimates. (c) cUFIR (dash-dotted), $\mathrm{cKF}$ (bold), and $\mathrm{cH}_{\infty}$ (dashed line) estimates.

We will perform additional procedures to acquire the EMG envelope with smoother dips and peaks. For this purpose, we applied two estimators the p-shift batch optimal FIR (OFIR) estimator and Kalman filter to every estimation of the of used algorithms (KF, UFIR, $\mathrm{H}_{\infty}, \mathrm{cKF}$, cUFIR, $\mathrm{cH}_{\infty}$ ), both filters are extended for the operation of smoothing. The first of these operates as a smoother in sense $p<0$ to $|p|-\operatorname{lag}$ [22]. In the second case of KF smoothing, it used future measurements to obtain the state estimate. We used the Rauch-Tung-Striebel (RTS) smoother, which is a fixed-interval smoother. This algorithm is showed in [26].

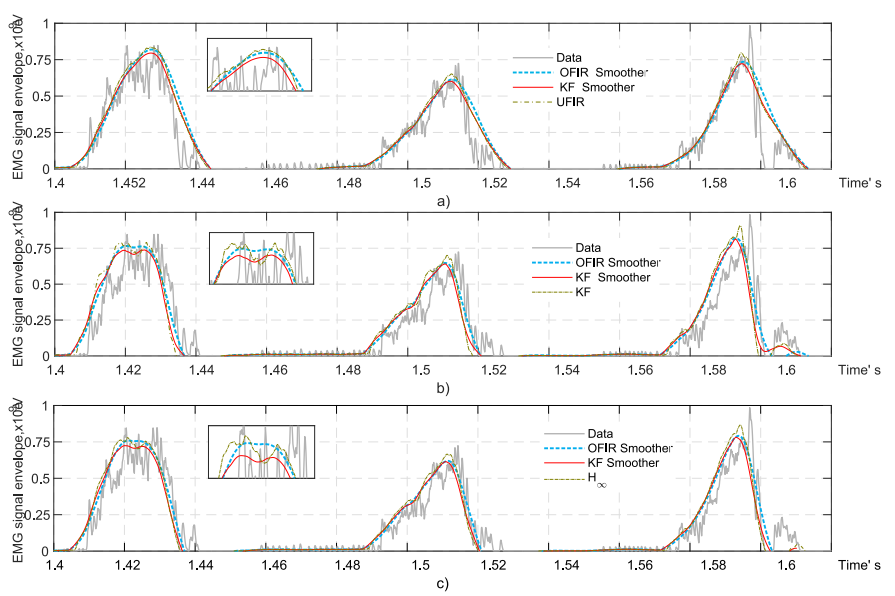

Fig. 3. A part of the EMG signal (1.4..1.6)s available from [13]: Envelope (data) obtained through the Hilbert transform (solid), OFIR Smoother (bold), KF Smoother (dash-dash), a) UFIR (solid), b) KF (Solid), and c) $\mathrm{H}_{\infty}$ (Solid) stimates.

From Fig. 3, the following can be noted. The smooth estimates of each of the algorithms. For optimum smooth we used $N_{\text {opt }}=140$ and $p=-10$.

\subsection{Test Gaussian Pulse}

Previous research has suggested that the EMG envelope must behave in a manner (distribution) by the activity of the signal, we know that given its power spectrum of a single motor unit firing with a mean rate of 15 spikes per second has a Gaussian envelope [23]. In this process, a Gaussian-shaped is present in EMG envelope distribution, where the parameters of interest are time, duration, and amplitude of the phases of activity. Gaussian is not the only shape of the EMG signal envelope to applications [24].

The EMG envelope given by each estimator has an approximation to the Gaussian pulse very significant. In Fig. 4, the Gaussian pulse is positioned to provide the best MSE sense adjustment for each algorithm in the "S1_A1_E1" signal.

TABLE I

Test Gaussian Pulse

\begin{tabular}{lcll}
\hline \multicolumn{3}{c}{ RMSE } \\
\hline & Estimating & OFIR Smoothing & KF Smoothing \\
\hline KF & 0.03149 & 0.02684 & 0.2413 \\
UFIR & 0.0313 & 0.02199 & 0.02388 \\
$H_{\infty}$ & 0.04068 & 0.022463 & 0.02906 \\
cKF & 0.0276 & 0.0198 & 0.02109 \\
cUFIR & 0.02623 & 0.01968 & 0.0212 \\
$\mathrm{c} H_{\infty}$ & 0.2885 & 0.02184 & 0.02214 \\
\hline
\end{tabular}



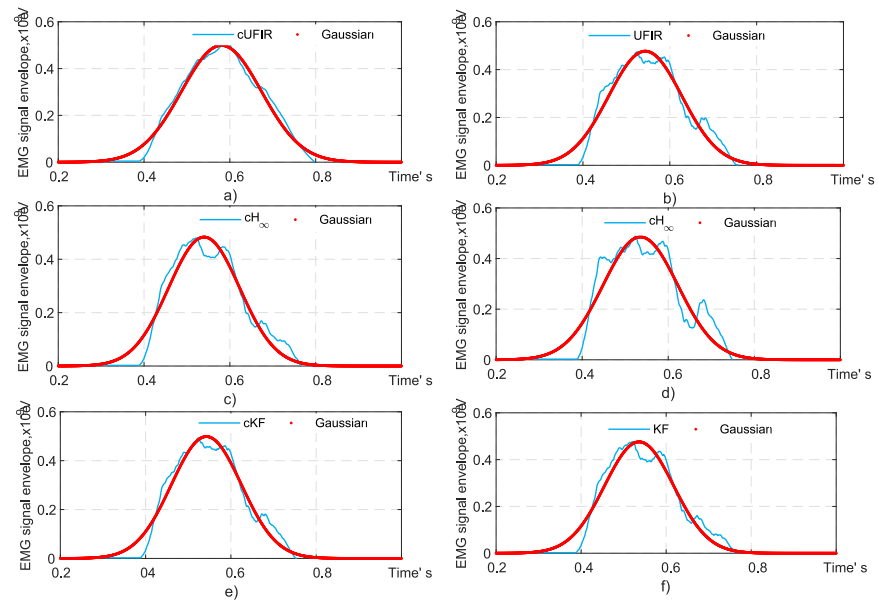

Fig. 4. Testing the envelope extracted using the algorithms by the gaussian in the MSE sense. Envelope extracted using (a) cUFIR, (b) UFIR, (c) $\mathrm{cH}_{\infty}$, (d) $\mathrm{H}_{\infty},(\mathbf{e}) \mathrm{KF}$, (f) and (e) cKF.

The table I displays the accuracy of the estimation of each algorithm for calculating a Gaussian continuous distribution function. It is observed data before estimation updating and data after treatment. It can note that envelope smoothing generated the best fit for Gaussian approximation of the signal.

\subsection{Robot Arm Control Systems by Using EMG Characterization}

Biorobotic systems can be categorized from the EMG envelope, as shown in Seccion IV-B, EMG envelope has a nearly Gaussian spatial distribution according to it, the EMG envelope can be expressed in terms of 'amplitude', 'duration', or 'time'. Features are extracted from EMG standardized envelope signal to be characterized. Thus the election term is labeled with a certain action so that thresholds of activation are created. Next, each feature is associated to an actuator that was integrated into a robotic system, the purpose of this is of producing a desired mechanical response, understood as angular displacement $\alpha$.

The calculated window, used to process of EMG envelope, is delimited by a segment of length $L$ equal to $200 \mathrm{~ms} L 300 \mathrm{~ms}$, because of the MUAP morphology, due to it will typically have a duration of 5-15 ms and the range of MUAP amplitude varies from $1000 \mathrm{uV}(1 \mathrm{mV})$ to $2000 \mathrm{uV}(2 \mathrm{mV})$ [14]. and an overlap between windows from $25 \%$ to $50 \%$ of the length. Next, an Adjustment is applied to ensure the appropriate values in the envelope. In this manner, it is possible to observe and evaluate the EMG signal.

In Fig.5 is possible to observe three windows that evaluate the signal separated. Firstly, the EMG envelope is categorized. Secondly, the extracted features are labeled to develop a certain action. Finally, activation thresholds are created. In order to achieve the characterization it is necessary to standardize the envelope. A standardized envelope is required because the EMG envelope is not sufficient to understand and relate the EMG signal to the force that a muscle is exerting due to the amplitude of the EMG signal depends on the distance from

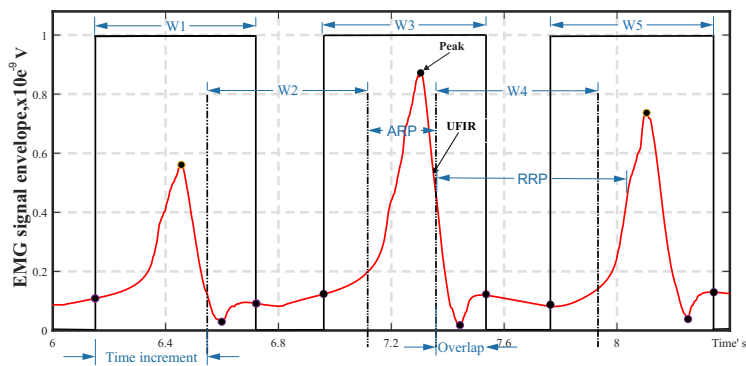

Fig. 5. The window used to calculate the EMG signal envelope and evaluate the signal separated.

the motor unit to the recording electrodes. In this way we can set the activation thresholds [15], [16]. The EMG envelope is categorized according to its amplitude and thus the labels are set for each action. The set angle has been calculated to provide a drive module by using the impedance model basis. Therefore, activation thresholds are obtained for four categories by using the standardized envelope [17].

\section{Control Results}

We used the muscular contraccions of the wrist to to achieve the control of $\Phi_{2}$, the elbow signal to represent $\Phi_{1}$, and the basic movements of the fingers to move the Creeper. Given the initial conditions, we integrate and find the positions of the joints by entering the values obtained from the characterization of the EMG envelope. Given $\dot{\Phi}$, we can observe the robot's trajectories to $\Phi_{1}=30^{\circ}, \Phi_{2}=120^{\circ}$, and the Creeper is forced to $0^{\circ}$, that creates the desired movement .

The objective is to get the minimum accumulated error and a minimum variation of angles in a specific time, given the joints position from an initial position to a desired position. The controller gives sudden speed changes to all diagnostics that occur during the test sequence. On this section can see the results of the speeds of joint 1 to $\Phi_{1}=30^{\circ}$ and the speed of joint 2 to $\Phi_{2}=90^{\circ}$, where, $\Phi_{2}$ has negative values. Therefore the angle variation of a joint between an instant of time and a time prior can be too wide.

The system error in response to the reference inputs for the estimate is given in Fig. 6. The benchmark targets were achieved by using PD control, we used values $k_{v}>0$ and $K_{p}>0$, for better stability of the system. Where $k_{v}$ is the differential constant and $K_{p}$ is the proportionality constant. The PD controller was implemented for the specified time to find rms error and thus obtain the cost function to improve the response of this controller and achieve better fit. It is noticeable that the error of the unfiltered envelope is quite high and it takes longer to achieve stability, due to EMG envelope raw produces a fast variation, such variations can then be observed in Fig.1 and Fig.2. With respect to the results obtained, the $\mathrm{KF}, \mathrm{H}_{\infty}$ filters had similar results as the UFIR, while cKF, $\mathrm{cH}_{\infty}$ showed a behavior given by the cUFIR error. It is fairly obvious that the tripping error given in the cUFIR is large. However, the error reaches zero in a shorter space of time than 
WSEAS TRANSACTIONS on BIOLOGY and BIOMEDICINE DOI: $10.37394 / 23208.2021 .18 .12$

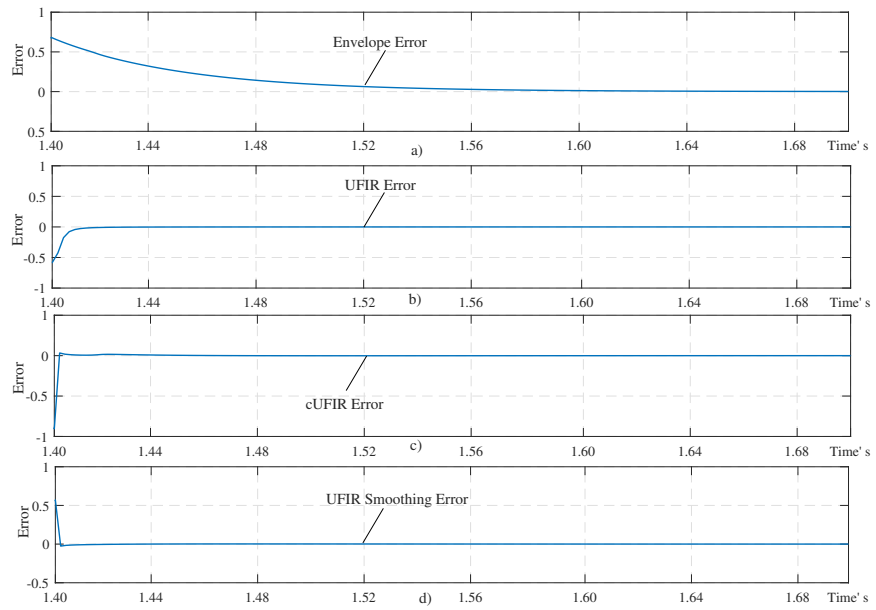

Fig. 6. Joint speeds: $\Phi_{1}$ speed is given by the solid line and $\Phi_{2}$ speed is given by the dash-dash.

in the UFIR filter, No matter that in the second the tripping error is less. Finally, we can see that the zero error is given much faster in the smoothed estimation. Trajectory tracking is not perfect, however, in regards to performance it does very well.

\section{Conclusions}

Applications of the modified cKF, $\mathrm{c} H_{\infty}$ filter, and cUFIR filter to the envelope extraction from the EMG signal data have shown their efficiency both in the envelope shaping and outliers detection. Better results were achieved by interpreting ripples in the EMG signal envelope as Gauss-Markov noise. Accordingly, it has been shown that the envelope extracted using the modified filters is much better smoothed and many artifacts can be removed from the EMG signal with high accuracy under the CMN assumption. Experimental verification provided based on different EMG signals have shown that the $\mathrm{cKF}, \mathrm{cH}_{\infty}$, and cUFIR algorithms are efficient when the MUAP density is low, in which case intensive excursions in the envelope are reminiscent of the colored noise. Shaped with high a MUAP density, the envelope typically demonstrates smaller variations and the filters designed become less efficient. In both cases, the color factor must be optimized to approach the desired envelope in the best way. The high variation could cause physical harm and instability in the system, this is where the theory of control is applied. Now, we may say that in the time of the estimation of the filters for their establishment is fast and the error in stationary state can be almost non-existent.

\section{References}

[1] E. Orosco, J, Gaia Amoros, J. Gimenez, C. Soria, "Deep learning-based classification using Cumulants and Bispectrum of EMG signals," IEEE Latin America Trans., vol. 17, no. 12, pp. 1946-1953, 2019.

[2] G. Lu, J. Brittain, P. Holland, J. Yianni, et al, "Removing ECG noise from surface EMG signals using adaptive filtering," Neuroscience Lett., vol. 462, no. 1, pp. 14-19, 2009.
Sandra Marquez-Figueroa,

Yuriy S. Shmaliy, Oscar Ibarra-Manzano
[3] S. Marquez-Figueroa, Y. S. Shmaliy, and O. Ibarra-Manzano, "Optimal extraction of EMG signal envelope and artifacts removal assuming colored measurement noise, Biomed. Signal Process. Contr, vol. 57, 101679, pp. 1-8, Mar. 2020

[4] S. Choi, S. Han, D. Jung, H. Hwang, C. Lim, et al, "Highly conductive, stretchable and biocompatible $\mathrm{Ag}-\mathrm{Au}$ core-sheath nanowire composite for wearable and implantable bioelectronics," Nat. Nanotech., vol. 13 , pp. 1048-1056, 2018.

[5] Schneider, J., Gründig, B., Renneberg, R., Cammann, K., Madaras, M. B., Buck, R. P., Vorlop, K.-D. (1996). Hydrogel matrix for three enzyme entrapment in creatine/creatinine amperometric biosensing. Analytica Chimica Acta, 325(3), 161-167.

[6] L. Han, X. Lu, M. Wang, D. Gan, et al, "A Mussel-inspired conductive, self-adhesive, and self-healable tough hydrogel as cell stimulators and implantable bioelectronics, Small, vol. 13, no. 2, 1601916, 2016.

[7] H. Hasan, M. H. Safdar, S. Zahid, M. Bibi, and A. Gul, "Robotics and High-Throughput Techniques,' Omics Technologies and BioEngineering, VOL.1, NO. 1, PP. 155-166, 2018.

[8] T. D'Alessio and S. Conforto, "Extraction of the envelope from surface EMG signals," IEEE Eng. Med. Biol. Mag., vol. 20, no. 6, pp. 55-61, 2001.

[9] L. Chen and H. Yaru, "Feature extraction and classification of ehgbetween pregnancy and labour group using hilbert-huang transform andextreme learning machine," Comp Math Method Med, vol. 2017, pp. 1-9, Feb. 2017.

[10] R. Kleissen and G. Zilvold, "Estimation uncertainty in ensemble averagesurface emg profiles during gait," J. Electromyog. Kinesiology, vol. 4, pp. 83-94, 1994.

[11] I. Stirn, T. Jarm, Tomaz, V. Peter Kapus, and V. Strojnik, "Evaluation of mean power spectral frequency of EMG signal during 100 metre crawl," Europ. J. Sport Sci., vol. 13, pp. 1-10, 2011.

[12] Y. Chien Hung, H. Wen Vincent Young, C. Yen Wang, et al, "Quantifying spasticity with limited swinging cycles using pendulum test based onphase amplitude coupling," IEEE Trans. Nucl. Sci, vol. 24, pp. 1-1, 2016.

[13] López R, Torres J, Salazar S, et al. Desarrollo de un Exoesqueleto para Rehabilitación de Tobillo y Rodilla. Rev Mex Ing Biomed. 2014;35(1):13-28

[14] D. Preston and B. Shapiro, "Needle electromyography," Neurologic Clinics, vol. 20, no. 2, pp. 361-396, 2002.

[15] M. Aminoff, Electromyography in clinical practice. Churchill Livingstone.(1987).

[16] A. Guyton, Tratado de Fisiología Médica, Interamericana, Madrid, 1982.

[17] A. Furui, S. Eto, K. Nakagaki, et al, "A myoelectric prosthetic hand with muscle synergy-based motion determination and impedance model-based biomimetic control," Science Robotics, vol. 4, no. 31, elocation-id = eaaw6339, 2019.

[18] Y. S. Shmaliy, "Unbiased FIR filtering of discrete time polynomial state space models," IEEE Trans. on Signal Process., vol. 57, no. 4, pp. 12411249, 2009.

[19] S. Marquez-Figueroa, Y. S. Shmaliy, and O. Ibarra-Manzano, "Optimal extraction of EMG signal envelope and artifacts removal assuming colored measurement noise," Biomed. Signal Process. Control, vol. 57, 101679, pp. 1-8, Mar. 2020

[20] D. Simon, Optimal State Estimaiton: Kalman, $H_{\infty}$, and Nonlinear Approaches, John Wiley \& Sons, Hoboken, NJ, 2006.

[21] Y. S. Shmaliy, F. Lehmann, S. Zhao, and C. K. Ahn, "Comparing robustness of the kalman, $H_{\infty}$, and UFIR filters," IEEE Trans. Signal Process., vol. 66, no. 13, pp. 3447-3458, 2018.

[22] Y. S. Shmaliy, "An iterative Kalman-like algorithm ignoring noise and initial conditions," IEEE Trans. Signal Process., vol. 59, pp. 2465-2473, 2011.

[23] J.-J. Chen, R. G. Shiavi, and L.-Q. Zhang, "A quantitative and qualitative description of electromyographic linear envelopes for synergy analysis," IEEE Trans. Biomed. Eng., vol. 39, no. 1, pp. 9-18, Jan. 1992.

[24] Y. Du, H. Wang, S. Qiu, W. Yao, P. Xie and X. Chen, "An Advanced Adaptive Control of Lower Limb Rehabilitation Robot", Frontiers in Robotics and AI ., vol.5,pp.116,2018.

[25] Atzori, M., Gijsberts, A., Castellini, C. et al. Electromyography data for non-invasive naturally-controlled robotic hand prostheses. Sci Data 1 , 140053 (2014).

[26] D. Simon and Y. S. Shmaliy, "Unified forms for Kalman and finite impulse response filtering and smoothing," Automatica, vol. 49, no. 6, pp. 1892-1899, Jun. 2013. 


\section{Creative Commons Attribution License 4.0 (Attribution 4.0 International, CC BY 4.0)}

This article is published under the terms of the Creative Commons Attribution License 4.0

https://creativecommons.org/licenses/by/4.0/deed.en_US 